\title{
Factors Causing Overbudget for Roof Cover Work in the Construction of High-rise Buildings
}

\author{
Rizki Andriani ${ }^{1}$ and Budi Susetyo ${ }^{2}$ \\ ${ }^{1}$ Departement Master Program of Civil Engineering \\ Mercu Buana University \\ Jakarta Indonesia \\ ${ }^{2}$ Departement Master Program of Civil Engineering \\ Mercu Buana University \\ Jakarta Indonesia
}

\begin{abstract}
The development of building construction in Indonesia is experiencing rapid development. In 2018, the total construction projects are predicted to increase by 3\% compared to 2017. The concept of building high-rise buildings now uses the concept of green building. Where in the use of materials must be according to the approval of a green building consultant. This study uses roof covering material with solar panels, using these materials project planning becomes overbudget. Overbudget that occurs when planning with the green building concept is $15 \%$ of the initial contract. Therefore, the researcher will discuss the factors that influence the cost efficiency of the roof cover work of high-rise buildings. Processing data using SPSS tools (Statistical Package for Social Sciences). From this data processor with SPSS, there were 10 factors that caused the overbudget of roof cover work on the construction of high-rise buildings using wrong estimation techniques, complicated development requirements, inadequate project funding, not taking into account unexpected costs, inaccurate estimation of costs, data and incomplete project information, material selection according to work drawings, owner changes to design changes when construction is underway, lack of coordination between construction manager - planner and estimated cost of building high-rise buildings. For this reason, look for factors that cause roof cover work in the construction of high-rise buildings.
\end{abstract}

Key Words: Key Success Factors, Overbudget, Solar Panels, High-rise Buildings.

\section{INTRODUCTION}

Multi-storey buildings are buildings that have more than one floor vertically. This study will discuss roof cover work in high-rise academic buildings. The roof is a part of a building that functions as a cover for all the rooms below. The roof is also a crown that has a function to add beauty and to protect buildings from heat and rain. In the current development, roof cover material is increasingly developing roof cover using solar panels. Solar panels are technologies that function to convert or convert solar radiation into electrical energy directly. There are 2 types of solar panels, namely solar panels using batteries and not using batteries.

\section{ESTIMATED COSTS}

Cost estimation is the process of estimating the relationship between costs and the effect of these costs. Estimated cost is divided estimation of direct costs and estimates of indirect costs. Estimated direct costs are calculated based on the multiplication of bid unit prices with the volume of work referring to the drawings and technical specifications, while the estimated indirect costs are not easy because there is no accurate reference of information as well as the drawings and technical specifications [1], According to [2], cost estimation is an iterative process of developing estimates of the monetary resources needed to complete project activities. According to [3] estimated costs are areas of engineering activities where experience and technical considerations are used in applying science especially the problem of estimating costs and controlling costs [4] 


\section{EFFICIENT COSTS}

Cost efficiency is a strategic choice used by many companies, especially companies engaged in construction. Efficient costs on construction projects are usually carried out in material selection where using material with the same function is used material at a cheaper price and still in the required standard specifications. Efficient project costs are usually controlled by the project manager, cost control and Quantity Surveyor. For this reason, material selection definitely requires approval from that party.

\section{PROJECT OVERBUDGET}

Freezing costs is the construction cost of a project which, during the implementation phase, exceeds (budget) the project budget set at the beginning (estimated cost), thus causing significant losses to the contractor [5]. Project overbudget occurs in a construction project can be caused by internal parties or external parties from the construction project itself. The cost swelling itself is divided into three parts, namely: 1. Freezing costs in the initial stages of construction, 2. Freezing costs during the construction project process, and Freezing post-construction costs. Therefore, in this study we will discuss the factors that influence the overbudget of construction projects.

\section{RESEARCH METHOD}

According to research methods [6] is basically a scientific way to get valid data with objectives that are discovery, proof and development of a knowledge so that the results can be used to understand, solve and anticipate problems. The flow in this study is:

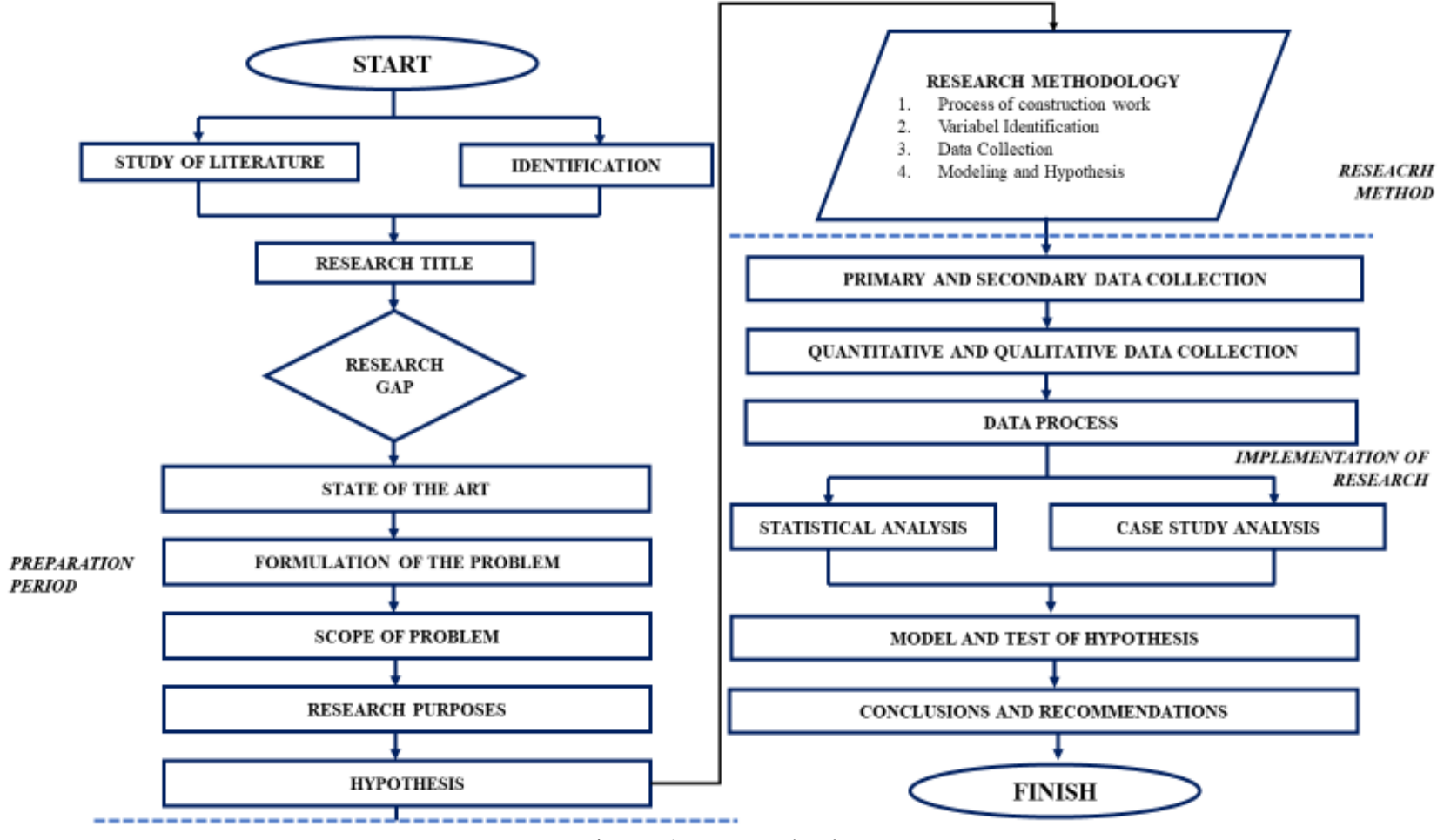

Figure 1. Research Flow

From the research method above, it can be concluded that the flow of this study uses successful key factors obtained from reference to journals, books or e-books. The results of the key success factors can be done by analyzing the data which then can draw conclusions from the efficiency and effectiveness in terms of costs that can improve the project's overbudget.

\section{IDENTIFICATION OF VARIABLES}

This study has 3 independent variables and 1 dependent variable for processing and analyzing the results of factors in roofing work of high-rise buildings, variables can be in the form of theory or object of research [7]. These variables are:

- Dependent Variabel : 1. Overbudget (X1)

2. Design of high-rise building (X2)

3. Roof cover work (X3)

- Independent Variabel : - Cost performance (Y1)

Variable identification is obtained from the key success factors obtained from journals, books, e-books and other references 
respondents obtained from this study were 52 respondents. Determination of the number of respondents using the Slovin method. Where to use the target population, the target population is the number of respondents according to the project organizational structure. The following are influential factors:

Table 1. Factors that Influence Overbudget Roof Cover Work

\begin{tabular}{|c|c|c|c|c|}
\hline NO & \multicolumn{2}{|c|}{ MAIN FACTOR } & SUBFACTOR & \multirow{2}{*}{$\begin{array}{l}\text { KEY SUCCESS FACTOR } \\
\text { Use the wrong estimation technique }\end{array}$} \\
\hline 1 & \multirow{16}{*}{$\mathbf{X} 1$} & $\mathrm{X} 1-1$ & \multirow{16}{*}{ OVERBUDGET } & \\
\hline 2 & & $\mathrm{X} 1-2$ & & Complex development requirements \\
\hline 3 & & $X 1-3$ & & Inaccurate estimated costs \\
\hline 4 & & $X 1-4$ & & Project data and information are incomplete \\
\hline 5 & & $\mathrm{X} 1-5$ & & $\begin{array}{l}\text { Lack of coordination between contractor } \\
\text { construction manager planners }\end{array}$ \\
\hline 6 & & $\mathrm{X} 1-6$ & & Design drawings and working drawings \\
\hline 7 & & $\mathrm{X} 1-7$ & & Labor productivity \\
\hline 8 & & $\mathrm{X} 1-8$ & & Poor cost control in the field \\
\hline 9 & & $X 1-9$ & & High equipment prices / rental \\
\hline 10 & & $\mathrm{X} 1-10$ & & Error managing equipment storage \\
\hline 11 & & $\mathrm{X} 1-11$ & & Lack of construction materials \\
\hline 12 & & $\mathrm{X} 1-12$ & & Unhealthy competition \\
\hline 13 & & $\mathrm{X} 1-13$ & & Too many projects are handled at the same time \\
\hline 14 & & $\mathrm{X} 1-14$ & & Consultants are less able to supervise the project \\
\hline 15 & & $\mathrm{X} 1-15$ & & Poor quality control \\
\hline 16 & & $\mathrm{X} 1-16$ & & There is an increase in material prices \\
\hline 17 & \multirow{8}{*}{$\mathbf{X} 2$} & $\mathrm{X} 2-1$ & \multirow{8}{*}{$\begin{array}{c}\text { HIGH-LEVEL } \\
\text { BUILDING } \\
\text { DESIGN }\end{array}$} & Classification of High-rise Buildings \\
\hline 18 & & $\mathrm{X} 2-2$ & & Design and Planning of High-rise Buildings \\
\hline 19 & & $\mathrm{X} 2-3$ & & Control of High-rise Buildings \\
\hline 20 & & $\mathrm{X} 2-4$ & & Operations and Activities of High-rise Buildings \\
\hline 21 & & $\mathrm{X} 2-5$ & & Team Performance of High-rise Buildings \\
\hline 22 & & $\mathrm{X} 2-6$ & & Project Optimization \\
\hline 23 & & $\mathrm{X} 2-7$ & & Estimated Cost of Building High-rise Buildings \\
\hline 24 & & $\mathrm{X} 2-8$ & & Installation Process and Work Technique \\
\hline 25 & \multirow{9}{*}{$\mathbf{X 3}$} & $\mathrm{X} 3-1$ & \multirow{9}{*}{$\begin{array}{c}\text { ROOF } \\
\text { CLOSING } \\
\text { WORK }\end{array}$} & Design drawings \\
\hline 26 & & $\mathrm{X} 3-2$ & & $\begin{array}{l}\text { Material Selection In Accordance With Working } \\
\text { Pictures }\end{array}$ \\
\hline 27 & & X3-3 & & Conformity and Clarity of Specifications \\
\hline 28 & & $\mathrm{X} 3-4$ & & Availability of Material Stock \\
\hline 29 & & $\mathrm{X} 3-5$ & & $\begin{array}{l}\text { Roofing Materials and their Applications in Building } \\
\text { Construction }\end{array}$ \\
\hline 30 & & X3-6 & & Expertise and Experience in Work \\
\hline 31 & & $\mathrm{X} 3-7$ & & Accuracy (Determination) and Measurement \\
\hline 32 & & $\mathrm{X} 3-8$ & & Aesthetic and Appearance Considerations \\
\hline 33 & & X3-9 & & Material Costs and Equipment used \\
\hline 34 & \multirow{5}{*}{ Y1 } & Y1-1 & \multirow{5}{*}{ COST } & Inadequate project funding \\
\hline 35 & & $\mathrm{Y} 1-2$ & & Does not take into account unexpected costs \\
\hline 36 & & Y1-3 & & $\begin{array}{l}\text { The owner made a design change when the } \\
\text { construction was running }\end{array}$ \\
\hline 37 & & $\mathrm{Y} 1-4$ & & Planning is not competent \\
\hline 38 & & Y1-5 & & Miscommunication in design planning \\
\hline
\end{tabular}




\section{PROCESSING STAGE}

The research method determines how a research process is carried out from data collection, processing data into information to be analyzed and finally producing findings that can be drawn conclusions. The steps in this study are as follows: 1 . Deciphering the background, 2. Identifying the problem, 3 Arranging the theoretical foundation, 4. Determining the research variables, 5. Choosing research instruments, 6. Determining the subject of research, 7. Collecting data, 8. Processing data and 9. Writing research report. [8]

In its own validity test [9] a measure that can show the validity or validity of the instrument. So in testing the validity it refers to an instrument in carrying out its functions. Variables obtained from journals, e-books, and books that can be published. The process of testing the validity and reliability is carried out using the tools in the SPSS program as follows: data processing flow:

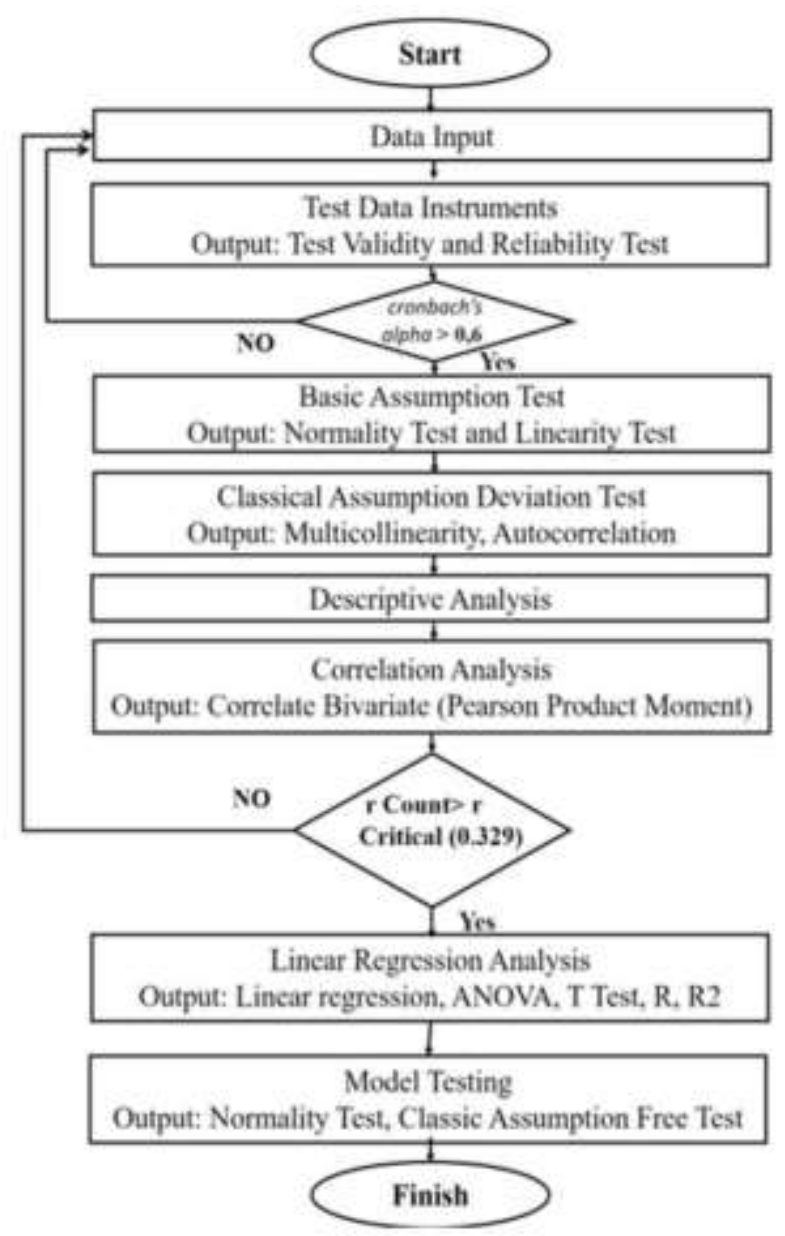

Figure 2. Flow diagram

\section{DETERMINATION OF THE NUMBER OF RESPONDENTS}

This study uses a target population type, where questionnaires will be distributed to people directly involved in the construction of the high-rise building. Determination of the number of respondents using Slovin formula. the number of targer population is $\mathrm{N}=$ 60 people. The following is the number of samples using the Slovin method:

$n=\frac{N}{N x d^{2}+1}$

$n=\frac{60}{60 x 0.05^{2}+1}=\mathbf{5 2 , 1 7} \sim \mathbf{5 2}$ Respondents

So, in this study there were 52 number of respondents. 
International Journal of Engineering Research And Advanced Technology, Vol.5, Issue 5, May-2019

\section{VALIDITY AND RELIABILITY TEST}

Judging from the value of corrected item total correlation if the value is more than 0.300 , it can be stated Relatively and the value of Cronbach's Alpha (Calculated Validity).

Table 2. Reliability Test and Validity Test X1

\begin{tabular}{|c|c|c|c|c|c|c|}
\hline VARIABLE X1 & $\begin{array}{c}\text { RELIABILITY } \\
\text { CALCULATED } \\
\text { VALUE }\end{array}$ & $\begin{array}{c}\text { RELIABILITY } \\
\text { STANDARD } \\
\text { VALUE }\end{array}$ & $\begin{array}{l}\text { RELIABLE/NON } \\
\text { RELIABLE }\end{array}$ & $\begin{array}{c}\text { VALUE OF } \\
\text { VALIDITY } \\
\text { COUNT }\end{array}$ & $\begin{array}{c}\text { STANDARD } \\
\text { VALIDITY } \\
\text { VALUE }\end{array}$ & $\begin{array}{c}\text { VALID/NON } \\
\text { VALID }\end{array}$ \\
\hline $\mathrm{X} 1-1$ & 0,619 & 0,300 & RELIABEL & 0,898 & 0,600 & VALID \\
\hline VARIABLE X1 & $\begin{array}{l}\text { RELIABILITY } \\
\text { CALCULATED } \\
\text { VALUE }\end{array}$ & $\begin{array}{l}\text { RELIABILITY } \\
\text { STANDARD } \\
\text { VALUE }\end{array}$ & $\begin{array}{l}\text { RELIABLE/NON } \\
\text { RELIABLE }\end{array}$ & $\begin{array}{c}\text { VALUE OF } \\
\text { VALIDITY } \\
\text { COUNT }\end{array}$ & $\begin{array}{l}\text { STANDARD } \\
\text { VALIDITY } \\
\text { VALUE }\end{array}$ & $\begin{array}{c}\text { VALID/NON } \\
\text { VALID }\end{array}$ \\
\hline $\mathrm{X} 1-2$ & 0,289 & 0,300 & NON RELIABEL & 0,910 & 0,600 & VALID \\
\hline $\mathrm{X} 1-3$ & 0,554 & 0,300 & RELIABEL & 0,900 & 0,600 & VALID \\
\hline $\mathrm{X} 1-4$ & 0,496 & 0,300 & RELIABEL & 0,902 & 0,600 & VALID \\
\hline$\times 1-5$ & 0,454 & 0,300 & RELIABEL & 0,903 & 0,600 & VALID \\
\hline$\times 1-6$ & 0,434 & 0,300 & RELIABEL & 0,904 & 0,600 & VALID \\
\hline $\mathrm{X} 1-7$ & 0,388 & 0,300 & RELIABEL & 0,905 & 0,600 & VALID \\
\hline $\mathrm{X} 1-8$ & 0,425 & 0,300 & RELIABEL & 0,904 & 0,600 & VALID \\
\hline$X 1-9$ & 0,727 & 0,300 & RELIABEL & 0,894 & 0,600 & VALID \\
\hline $\mathrm{X} 1-10$ & 0,671 & 0,300 & RELIABEL & 0,896 & 0,600 & VALID \\
\hline X1-11 & 0,701 & 0,300 & RELIABEL & 0,895 & 0,600 & VALID \\
\hline $\mathrm{X} 1-12$ & 0,639 & 0,300 & RELIABEL & 0,897 & 0,600 & VALID \\
\hline $\mathrm{X} 1-13$ & 0,842 & 0,300 & RELIABEL & 0,890 & 0,600 & VALID \\
\hline X1-14 & 0,715 & 0,300 & RELIABEL & 0,895 & 0,600 & VALID \\
\hline $\mathrm{X} 1-15$ & 0,644 & 0,300 & RELIABEL & 0,897 & 0,600 & VALID \\
\hline $\mathrm{X} 1-16$ & 0,700 & 0,300 & RELIABEL & 0,895 & 0,600 & VALID \\
\hline
\end{tabular}

From table 2. Test reliability and validity test can be concluded for the count reliability test more than 0.300 then the results are reliable and if the test validity count is more than 600 then the result is valid

Table 3. Test Reliability and Test Validity X2

\begin{tabular}{|c|c|c|c|c|c|c|}
\hline VARIABLE X2 & $\begin{array}{c}\text { RELIABILITY } \\
\text { CALCULATED } \\
\text { VALUE }\end{array}$ & $\begin{array}{c}\text { RELIABILITY } \\
\text { STANDARD } \\
\text { VALUE }\end{array}$ & $\begin{array}{c}\text { RELIABLE/NON } \\
\text { RELIABLE }\end{array}$ & $\begin{array}{c}\text { VALUE OF } \\
\text { VALIDITY } \\
\text { COUNT }\end{array}$ & $\begin{array}{c}\text { STANDARD } \\
\text { VALIDITY } \\
\text { VALUE }\end{array}$ & $\begin{array}{c}\text { VALID/NON } \\
\text { VALID }\end{array}$ \\
\hline $\mathrm{X} 2-1$ & 0,528 & 0,300 & RELIABEL & 0,823 & 0,600 & VALID \\
\hline$X 2-2$ & 0,580 & 0,300 & RELIABEL & 0,816 & 0,600 & VALID \\
\hline$X 2-3$ & 0,810 & 0,300 & RELIABEL & 0,783 & 0,600 & VALID \\
\hline$X 2-4$ & 0,440 & 0,300 & RELIABEL & 0,834 & 0,600 & VALID \\
\hline$X 2-5$ & 0,530 & 0,300 & RELIABEL & 0,823 & 0,600 & VALID \\
\hline$X 2-6$ & 0,544 & 0,300 & RELIABEL & 0,821 & 0,600 & VALID \\
\hline$X 2-7$ & 0,690 & 0,300 & RELIABEL & 0,804 & 0,600 & VALID \\
\hline$X 2-8$ & 0,439 & 0,300 & RELIABEL & 0,834 & 0,600 & VALID \\
\hline
\end{tabular}

From table 3 . Test reliability and validity test can be concluded for the count reliability test more than 0.300 then the results are reliable and if the test validity count is more than 600 then the result is valid 
International Journal of Engineering Research And Advanced Technology, Vol.5, Issue 5, May-2019

Table 4. Test Reliability and Validity Test X3

\begin{tabular}{|c|c|c|c|c|c|c|}
\hline VARIABLE X3 & $\begin{array}{c}\text { RELIABILITY } \\
\text { CALCULATED } \\
\text { VALUE }\end{array}$ & $\begin{array}{c}\text { RELIABILITY } \\
\text { STANDARD } \\
\text { VALUE }\end{array}$ & $\begin{array}{c}\text { RELIABLE/NON } \\
\text { RELIABLE }\end{array}$ & $\begin{array}{c}\text { VALUE OF } \\
\text { VALIDITY } \\
\text { COUNT }\end{array}$ & $\begin{array}{c}\text { STANDARD } \\
\text { VALIDITY } \\
\text { VALUE }\end{array}$ & $\begin{array}{c}\text { VALID/NON } \\
\text { VALID }\end{array}$ \\
\hline X3-1 & 0,307 & 0,300 & RELIABEL & 0,7296 & 0,600 & VALID \\
\hline X3-2 & 0,241 & 0,300 & NON RELIBEL & 0,7381 & 0,600 & VALID \\
\hline X3-3 & 0,487 & 0,300 & RELIABEL & 0,6986 & 0,600 & VALID \\
\hline X3-4 & $-0,013$ & 0,300 & NON RELIBEL & 0,7731 & 0,600 & VALID \\
\hline X3-5 & 0,452 & 0,300 & RELIABEL & 0,7051 & 0,600 & VALID \\
\hline X3-6 & 0,617 & 0,300 & RELIABEL & 0,6740 & 0,600 & VALID \\
\hline X3-7 & 0,169 & 0,300 & NON RELIBEL & 0,7528 & 0,600 & VALID \\
\hline X3-8 & 0,715 & 0,300 & RELIABEL & 0,6556 & 0,600 & VALID \\
\hline X3-9 & 0,758 & 0,300 & RELIABEL & 0,6448 & 0,600 & VALID \\
\hline
\end{tabular}

From table 4. Test reliability and validity test can be concluded for the count reliability test of more than 0.300 then the results are reliable and if the test validity count is more than 600 then the result is valid

Table 5. Reliability and Validity Test Y1

\begin{tabular}{|c|c|c|c|c|c|c|}
\hline $\begin{array}{c}\text { VARIABLE } \\
\text { Y1 }\end{array}$ & $\begin{array}{c}\text { RELIABILITY } \\
\text { CALCULATED } \\
\text { VALUE }\end{array}$ & $\begin{array}{c}\text { RELIABILITY } \\
\text { STANDARD } \\
\text { VALUE }\end{array}$ & $\begin{array}{c}\text { RELIABLE/NON } \\
\text { RELIABLE }\end{array}$ & $\begin{array}{c}\text { VALUE OF } \\
\text { VALIDITY } \\
\text { COUNT }\end{array}$ & $\begin{array}{c}\text { STANDARD } \\
\text { VALIDITY } \\
\text { VALUE }\end{array}$ & $\begin{array}{c}\text { VALID/NON } \\
\text { VALID }\end{array}$ \\
\hline Y1-1 & 0,414 & 0,300 & RELIABEL & 0,639 & 0,600 & VALID \\
\hline Y1-2 & 0,206 & 0,300 & RELIABEL & 0,711 & 0,600 & VALID \\
\hline Y1-3 & 0,427 & 0,300 & RELIABEL & 0,632 & 0,600 & VALID \\
\hline Y1-4 & 0,634 & 0,300 & RELIABEL & 0,524 & 0,600 & VALID \\
\hline Y1-5 & 0,497 & 0,300 & RELIABEL & 0,600 & 0,600 & VALID \\
\hline
\end{tabular}

After obtaining a variable that is declared to be reliable and valid, the variable will then be analyzed by looking for the Mean value of each variable in the questionnaire. 
Table 6. Questionnaire Ranking Results

\begin{tabular}{|c|c|c|c|c|c|c|c|c|c|c|c|c|c|}
\hline \multirow{2}{*}{ RANK } & \multirow{2}{*}{$\begin{array}{l}\text { MAIN } \\
\text { FACTO }\end{array}$} & \multirow{2}{*}{$\begin{array}{c}\text { SUB } \\
\text { FACTOR }\end{array}$} & \multirow{2}{*}{ ITEM KSF } & \multicolumn{5}{|c|}{ RECAPITULATION OF QUESTIONARY } & \multirow{2}{*}{$\begin{array}{l}\text { AMOUNT OF } \\
\text { RESPONDENTS }\end{array}$} & \multirow{2}{*}{$\mathbf{w}$} & \multirow{2}{*}{$\mathbf{S}$} & \multirow{2}{*}{$\mathbf{R}$} & \multirow{2}{*}{ INDEX RII } \\
\hline & & & & 1 & 2 & 3 & 4 & 5 & & & & & \\
\hline 1 & 1 & $\mathrm{x} 1-1$ & $\begin{array}{l}\text { Use the wrong estimation } \\
\text { technique }\end{array}$ & 0 & 0 & 19 & 19 & 14 & 52 & 203 & 5 & 52 & 0,781 \\
\hline 2 & I & $\mathrm{X} 1-2$ & $\begin{array}{l}\text { Complex development } \\
\text { requirements }\end{array}$ & 0 & 0 & 20 & 19 & 13 & 52 & 201 & 5 & 52 & 0,773 \\
\hline 3 & IV & Y1-1 & Inadequate project funding & 0 & 0 & 21 & 18 & 13 & 52 & 200 & 5 & 52 & 0,769 \\
\hline 4 & IV & Y1-2 & $\begin{array}{l}\text { Does not take into account } \\
\text { unexpected costs }\end{array}$ & 0 & 0 & 21 & 19 & 12 & 52 & 199 & 5 & 52 & 0,765 \\
\hline 5 & 1 & $\mathrm{x} 1-3$ & Inaccurate estimated costs & 0 & 0 & 22 & 18 & 12 & 52 & 198 & 5 & 52 & 0,762 \\
\hline 6 & 1 & $\mathrm{X} 1-4$ & $\begin{array}{l}\text { Project data and information are } \\
\text { incomplete }\end{array}$ & 0 & 0 & 23 & 17 & 12 & 52 & 197 & 5 & 52 & 0,758 \\
\hline 7 & III & $x 3-2$ & $\begin{array}{l}\text { Material Selection In Accordance } \\
\text { With Working Pictures }\end{array}$ & 0 & 0 & 24 & 16 & 12 & 52 & 196 & 5 & 52 & 0,754 \\
\hline 8 & IV & Y1-3 & $\begin{array}{l}\text { The owner made a design change } \\
\text { when the construction was } \\
\text { running }\end{array}$ & 0 & 1 & 23 & 16 & 12 & 52 & 195 & 5 & 52 & 0,750 \\
\hline 9 & 1 & $\mathrm{x} 1-5$ & $\begin{array}{l}\text { Lack of coordination between } \\
\text { contractor construction manager } \\
\text { planners }\end{array}$ & 0 & 1 & 24 & 15 & 12 & 52 & 194 & 5 & 52 & 0,746 \\
\hline 10 & II & $x 2-7$ & $\begin{array}{l}\text { Estimated Cost of Construction of } \\
\text { High-rise Buildings }\end{array}$ & 0 & 1 & 25 & 14 & 12 & 52 & 193 & 5 & 52 & 0,742 \\
\hline 11 & I & $\mathrm{X} 1-6$ & $\begin{array}{l}\text { Design drawings and working } \\
\text { drawings }\end{array}$ & 0 & 2 & 24 & 14 & 12 & 52 & 192 & 5 & 52 & 0,738 \\
\hline 12 & III & X3-4 & Availability of Material Stock & 0 & 2 & 25 & 13 & 12 & 52 & 191 & 5 & 52 & 0,735 \\
\hline 13 & II & $x 2-1$ & Classification of High-rise Buildings & 0 & 2 & 25 & 14 & 11 & 52 & 190 & 5 & 52 & 0,731 \\
\hline 14 & 1 & $\mathrm{X} 1-7$ & Labor productivity & 0 & 2 & 26 & 13 & 11 & 52 & 189 & 5 & 52 & 0,727 \\
\hline 15 & II & $x 2-2$ & $\begin{array}{l}\text { Design and Planning of High-rise } \\
\text { Buildings }\end{array}$ & 0 & 2 & 27 & 12 & 11 & 52 & 188 & 5 & 52 & 0,723 \\
\hline 16 & III & $x 3-1$ & Design drawings & 0 & 2 & 28 & 11 & 11 & 52 & 187 & 5 & 52 & 0,719 \\
\hline 17 & 1 & $\mathrm{X} 1-8$ & Poor cost control in the field & 0 & 3 & 27 & 11 & 11 & 52 & 186 & 5 & 52 & 0,715 \\
\hline 18 & 1 & $\mathrm{x} 1-11$ & Lack of construction materials & 0 & 3 & 28 & 10 & 11 & 52 & 185 & 5 & 52 & 0,712 \\
\hline 19 & III & X3-5 & $\begin{array}{l}\text { Roofing Materials and their } \\
\text { Applications in Building } \\
\text { Construction }\end{array}$ & 0 & 4 & 27 & 10 & 11 & 52 & 184 & 5 & 52 & 0,708 \\
\hline 20 & III & X3-7 & $\begin{array}{l}\text { Accuracy (Determination) and } \\
\text { Measurement }\end{array}$ & 0 & 4 & 28 & 10 & 10 & 52 & 182 & 5 & 52 & 0,700 \\
\hline
\end{tabular}


International Journal of Engineering Research And Advanced Technology, Vol.5, Issue 5, May-2019

\begin{tabular}{|c|c|c|c|c|c|c|c|c|c|c|c|c|c|}
\hline \multirow{2}{*}{ RANK } & \multirow{2}{*}{$\begin{array}{r}\text { MAIN } \\
\text { FACTO } \\
\end{array}$} & \multirow{2}{*}{$\begin{array}{c}\text { SUB } \\
\text { FACTOR }\end{array}$} & \multirow{2}{*}{ ITEM KSF } & \multicolumn{5}{|c|}{ RECAPITULATION OF QUESTIONARY } & \multirow{2}{*}{$\begin{array}{c}\text { AMOUNT OF } \\
\text { RESPONDENTS }\end{array}$} & \multirow{2}{*}{ w } & \multirow{2}{*}{$\mathbf{S}$} & \multirow{2}{*}{$\mathbf{R}$} & \multirow{2}{*}{ INDEX RII } \\
\hline & & & & 1 & 2 & 3 & 4 & 5 & & & & & \\
\hline 21 & III & $\mathrm{x} 3-3$ & $\begin{array}{l}\text { Conformity and Clarity of } \\
\text { Specifications }\end{array}$ & 0 & 4 & 29 & 9 & 10 & 52 & 181 & 5 & 52 & 0,696 \\
\hline 22 & II & $x 2-4$ & $\begin{array}{l}\text { Operations and Activities of High- } \\
\text { rise Buildings }\end{array}$ & 0 & 4 & 29 & 10 & 9 & 52 & 180 & 5 & 52 & 0,692 \\
\hline 23 & II & $x 2-6$ & Project Optimization & 0 & 5 & 29 & 8 & 10 & 52 & 179 & 5 & 52 & 0,688 \\
\hline 24 & I & $x 1-12$ & Unhealthy competition & 0 & 5 & 29 & 9 & 9 & 52 & 178 & 5 & 52 & 0,685 \\
\hline 25 & III & X3-9 & $\begin{array}{l}\text { Material Costs and Equipment } \\
\text { used }\end{array}$ & 0 & 5 & 30 & 8 & 9 & 52 & 177 & 5 & 52 & 0,681 \\
\hline 26 & III & $x 3-8$ & $\begin{array}{l}\text { Aesthetic and Appearance } \\
\text { Considerations }\end{array}$ & 0 & 5 & 30 & 9 & 8 & 52 & 176 & 5 & 52 & 0,677 \\
\hline 27 & I & $x 1-13$ & $\begin{array}{l}\text { Too many projects are handled at } \\
\text { the same time }\end{array}$ & 0 & 6 & 29 & 9 & 8 & 52 & 175 & 5 & 52 & 0,673 \\
\hline 28 & 1 & X1-14 & $\begin{array}{l}\text { Consultants are less able to } \\
\text { supervise the project }\end{array}$ & 0 & 6 & 30 & 8 & 8 & 52 & 174 & 5 & 52 & 0,669 \\
\hline 29 & II & $x 2-3$ & Control of High-rise Buildings & 0 & 6 & 31 & 7 & 8 & 52 & 173 & 5 & 52 & 0,665 \\
\hline 30 & II & $x 2-5$ & $\begin{array}{l}\text { Team Performance of High-rise } \\
\text { Buildings }\end{array}$ & 0 & 7 & 30 & 7 & 8 & 52 & 172 & 5 & 52 & 0,662 \\
\hline 31 & 1 & $x 1-15$ & Poor quality control & 0 & 7 & 30 & 8 & 7 & 52 & 171 & 5 & 52 & 0,658 \\
\hline 32 & IV & Y1-4 & Planning is not competent & 0 & 7 & 31 & 7 & 7 & 52 & 170 & 5 & 52 & 0,654 \\
\hline 33 & IV & Y1-5 & $\begin{array}{l}\text { Miscommunication in design } \\
\text { planning }\end{array}$ & 0 & 8 & 30 & 7 & 7 & 52 & 169 & 5 & 52 & 0,650 \\
\hline 34 & III & $x 3-6$ & Expertise and Experience in Work & 0 & 8 & 31 & 6 & 7 & 52 & 168 & 5 & 52 & 0,646 \\
\hline 35 & II & $x 2-8$ & $\begin{array}{l}\text { Installation Process and Work } \\
\text { Technique }\end{array}$ & 0 & 8 & 31 & 7 & 6 & 52 & 167 & 5 & 52 & 0,642 \\
\hline 36 & I & $x 1-16$ & $\begin{array}{l}\text { There is an increase in material } \\
\text { prices }\end{array}$ & 0 & 9 & 30 & 7 & 6 & 52 & 166 & 5 & 52 & 0,638 \\
\hline 37 & I & $x 1-9$ & High equipment prices / rental & 1 & 9 & 29 & 6 & 7 & 52 & 165 & 5 & 52 & 0,635 \\
\hline 38 & I & $x 1-10$ & $\begin{array}{l}\text { Error managing equipment } \\
\text { storage }\end{array}$ & 1 & 9 & 29 & 7 & 6 & 52 & 164 & 5 & 52 & 0,631 \\
\hline
\end{tabular}

\section{CONCLUSION}

From the processing of SPSS data, it can be concluded that 10 work items are the factors that cause the overbudget of roof cover work in high-rise buildings:[10]

1. Using the wrong estimation technique

2. Complex development requirements

3. Inadequate project funding

4. Does not take into account unexpected costs

5. Inaccurate estimated costs

6. Project data and information are incomplete

7. Material selection according to the work picture

8. The owner made a design change when the construction was running

9. Lack of coordination between the construction-manager planner

10. Estimated cost of building a high-rise building

\section{REFERANCE}

[1] Phaobunjong, K. (2002). Parametric Cost Estimating Model for Conceptual Cost Estimating of Building Construction 
Projects. Dissertation, 408. Retrieved from https://repositories.lib.utexas.edu/handle/2152/845

[2] Schaufelberger, J. E., \& Holm, L. (2018). Cost estimating. In Management of Construction Projects. https://doi.org/10.4324/9781315529097-3

[3] AACE International Reccomended Practice No.17R97. 2012. Cost Estimate Classification Sistem.

[4] Soeharto, Imam. 1995. Manajemen Proyek Dari Konseptual Sampai Operasional. Jakarta : Erlangga

[5] Evans, M. (2005). Overdue and over budget, over and over again. The Economist.

[6] Phaobunjong, K. (2002). "Parametric Cost Estimating Model for Conceptual Cost Estimating of Building Construction Projects".

[7] Riduwan. (2004). Metode dan Teknik Menyusun Tesis. Cetakan Pertama. Bandung : Alfabeta CV

[8] Hardjomuljadi, Sarwono. 2014. Factor Analysis on Causal of Construction Claims and Disputes in Indonesia (with reference to the construction of hydroelectric power project in Indonesia). International Journal of Applied Engineering Research, ISSN 0973-4562, Volume 9, November 22, pp. 12421-12445

[9] Dysert, Larry.1999. "Developing a Parametric Model For Estimating Process Control Cost".

[10] Sugiyono. (2013). Metode Penelitian Kuantitatif, Kualitatif dan R\&D. Bandung : Alfabeta.CV 